\title{
A Paradigm Shift : Supply Chain Management 4.0 Triple "A" Method Agile, Anytime Anywhere, Always Visible
}

\author{
Alok Chandra
}

\begin{abstract}
Supply chain management (SCM) Strategy and design have innovate significantly in recent years. The buzzword currently when managing world-wide supply chains is design to growing(prenominal), incremental global complexity and volatility. Growing compression from fiscal nundinal and the impediment of increasing operating margins and working capital in this environment require efficient planning and execution of Supply Chain 4-0 "Supply Chain 4.0 - the relevancy of the Internet of Things, the application of sophisticated robotics, and the application of sophisticated analytics of big data in supply chain management: employment sensors in everything, Collaborative networks everywhere, automatize anything and everything, and analyse everything to significantly improve performance and customer satisfaction and available any time and from any where. Current paper is an effort to focus on the recent technological advances in supply chain management which is considered as Supply chain 4.0. The changes in ecosystem and benefits derived in in terms of efficiency, trackability, traceability, agility and managing bottom lines are significant.
\end{abstract}

Keywords : Supply Chain Strategy, IOT, Visibility ,Agility Traceability, Any time Anywhere.

\section{INTRODUCTION}

Over the last thirty years, supply line has experience a tremendous innovate: from a purely functional activity that narrate to sales or manufacturing and centred on catering to furnish of product lines and the distribution to customers, to an separate supply chain management activity. The concentrate of the supply chain management performance has change to sophisticated design procedure, such as analytical demand project or incorporate S\&OP, which have turn established business procedure in many corporation, while functional supply line has often been outsourced to third-party LSP

The some sort of murkiness and confusion frequently occurs in supply chain management, particularly in complex and variant-rich areas, with key stakeholders and decision-makers cut off from one another and potentially working at cross-purposes. Supply Chain 4.0 systems, with their emphasis on collecting mission critical data from sensors, have the potential to mitigate these

Revised Manuscript Received on July 22, 2019.

Dr Alok Chandra, Director, Centre of Excellence Supply Chain,

Universal Business School, Karjat, Mumbai, India sorts of decision-making siloes. With the new data being made available to them, manufacturers can make a push toward End-to-end (E2E) visibility. By making real-time data available throughout an entire organization, it is possible to illuminate the entire supply chain, giving stakeholders the power to make smarter, better informed decisions while boosting cross-operational planning and communication. Ultimately, businesses will be able to gain a more holistic, point-to-point view of their value chain and promote increasingly transparent manufacturing processes.

End to End Visibility E2E visibility is a noble goal for its own sake, but it can also help businesses bring about improvements that will radically change the Supply Chain .In particular, increased data visibility makes it possible for manufacturers to adopt intelligent planning solutions that respond to real-time inputs to demystify interrelated, variant-rich processes. When each value point from inbound stream to factory floor to customer delivery is digitized, made transparent, and more thoroughly understood, production sequences can be newly optimized, with areas of waste and inefficiency identified and remedied.

Agile Architecture An agile intelligent planning architecture should account for transport volumes, warehouse holdings, inventory levels, and potential disruptions and bottlenecks in order create production plans that are transparent, adaptable, and ultimately more cost-effective. If indeed we think of these new frontiers in the supply chain as a paradigm shift, practices like intelligent planning and smarter forecasting can offer supply chain professional not just new ways to improve processes, but a new way of looking at resources across all value points on the Supply Chain 4.0.

\section{INTERNET OF THINGS (IOT)}

A new paradigm shift enabled the introduction of various technologies in the supply chain market i.e the Internet of Things (IoT) into the entire supply chain ecosystem. This majorly emphasizes on the global 
networks of machines in smart factories capable of swapping information and controlling each other.

This cyber-physical system allows the smart factory to operate autonomously. For instance, a machine will automatically know the manufacturing process that needs to be applied to a product, along with the type of variation to be made to that product etc., so that the product is uniquely identified from the rest. The major impact of Supply Chain 4.0 stands when there is a transparent collaboration between suppliers, manufacturers, and customers in all the steps necessarily required. There is tremendous interest in how the Internet of Things (IoT) is transforming processes within and across organisations. And while there are multiple definitions floating around, IoT is about making things smarter so that they can talk to each other. This involves embedding sensors in the devices that then have the ability to collect and process the right data to generate useful insights about the end users and the value chain. IoT is fundamentally changing the definition of a product as it drives new business models that help companies better understand their end users and drive efficiencies across the value chain. Given its ability to impact both the top-line and bottom-line, IoT has rightfully caught the attention of the key executives of the industry.

Given the tremendous benefits anticipated by leveraging IoT, manufacturing and service organisations today have coined a variant to IoT that is specific to their universe. This is being termed the Industrial Internet-of-Things (IIoT) that encapsulates smart factories, extreme automation, industrial robots and more. IIOT again a function of the potential that the industry sees in IoT that IIoT has become an accepted it helps to differentiate it from the consumer IoT world, which is evolving as well. This new paradigm shift in leveraging IIoT is also called the fourth industrial revolution, known as Industrie 4.0 (German name for Industry 4.0), the term that the powerhouse of the manufacturing world Germany has coined.

Supply Chain 4.0 are already seeing the benefits of better productivity, reduced costs, higher customer satisfaction and a digitally-enabled workforce. The possibilities are endless with the only certainty being that supply chain tomorrow will be definitely different and smarter than what it was yesterday.

Industrial revolutions does not happen overnight. Everyone who has been active in logistics knows that innovations take especially long in the supply chain ecosystem. When we speak about digitization, we automatically picture a fully connected supply chain. Logistics objects, assets, humans and they are all connected with each other. The complete supply chain is transparent and agile. Constantly increasing customer expectations and short product lifecycles set the pace.

For example, A few years ago, Essentra Components had 300 machines producing 1.5 million mouldings a day at the Kidlington site. Today, there are 120 machines producing 3.7 million components a day. launched the S099 demand planning platform in EMEA and will shortly be launching this system in the Americas. This also supports aggregated global demand plan for all vendors, providing transparency across the entire business, improved response to the customer and ultimately a more efficient and dynamic manufacturing footprint

\section{TECHNOLOGICAL ADVANCEMENT}

Supply Chain 4.0 allows a space for technological advances in the traditional supply chain management process to cope with the disruptive changes in the market. The use of advanced analytics to enter the system to use data to its fullest potential proves no industry can survive this era if it has not moved towards the digital change. Advance technologies like IOT, Artificial Intelligence, Robotics support with the flexibility, transparency, and visibility necessary to respond to changes in planned production. Industries have started to look into the benefits provided by technologically advanced enterprises like IoT development companies, mobile app development companies etc. This ensures to maintain delivery timetables and continued movement of component parts throughout the value chain to reach to the end consumers. Supply Chain 4.0 provides automated technologies to various process that speeds up and eases the path of segments like job scheduling and management, inventory management, warehouse, and transportation strategy across the entire network.

Lighting Speed Supply chain process has evolved various new approaches to product distribution. This would reduce the delivery time, manage inventories without any loss in the process. Digitization has enabled the use of advanced forecasting approaches, such as predictive analytics of internal data (e.g., demand) and external data e.g., market trends etc., to make sure the consumers are supported with their demands and fulfilled before time. 
Example: In Amazon, products are shipped before the customer places an order. The customer order is later matched with a shipment that is already in the logistics network, and the shipment is rerouted to the exact customer destination.

Efficient Gone are the days when it was only a physical effort to bring down products to end consumers. With digitization, there is automation of both physical tasks and technology involvement that boosts supply-chain efficiency. Technologies like Robotics, AI, Machine advances are efficient in material handling, automating the process of receiving/unloading, to picking, packing, and even shipping of end products. Even transport has been upgraded with an autonomous transportation system for timely and non-failure delivery.

There are three key main enablers of transformation into digital supply chain: a clear strategy, new competencies, and an advanced environment. Digital supply chain starts with an understanding of the current operation digital waste, the lack of knowledge often leads to a failed road and thus it is important to encapsulate the technological advances. Capabilities regarding digitization then need to be created with planning. The final requirement is the implementation of an advanced technological approach. This altogether combines the advances that lead to digitally equipped innovative environment ready to penetrate any segment in and out and all for the end consumers.

Ok, it will take some time until we reach the truly digital supply chain. But most importantly, we also know from practical experience that logistics is a stressful business, especially for the staff involved. Powerful tools, IoT technology and real-time information are available. Actually, there is quite some room for improvement, above all regarding training and utilisation of resources.

Real-time IOT based Platform it is time for a paradigm shift in logistics from ERP-based document flow management to real-time IoT-based steering of the material flow. Obviously, the document flow in the ERP system remains important for what it was designed for: managing the business process, such as orders, confirmations, invoices. This remains the so called digital core. However, the time lag of document flow data compared to real-time material flow was and still is the main root cause for many logistic pain points.

This is exactly where pragmatic real-time logistics comes into play. Easy-to-implement IoT-based solutions that focus on added value and savings in the user story empower logistics staff to manage assets and objects in real time without system delays. This makes their work a whole lot easier and provides value-adding benefits. Apart from reducing wrong deliveries, logistics errors and costly countermeasures (e.g. express costs), current projects show that real-time logistics can:.

In daily work, uptime improvements of up to $30 \%$ do not come as a surprise: significantly fewer assets are needed for the same amount of transports. This, in turn, leads to a cutback of often oversized moving asset stock. Moreover, heatmaps can display hotspots and bottlenecks in the transportation flow. As soon as they are known, they can be counteracted, and targeted infrastructural improvements can be implemented.

Supply chains are extremely complex organisms, and no company has yet succeeded in building one that's truly digital. Indeed, many of the applications required are not yet widely used. But this will change radically over the next five to 10 years, with different industries implementing DSC at varying speeds. Companies that get there first will gain a difficult-to-challenge advantage in the race to Supply Chain 4.0 and will be able to set, or at least influence, technical standards for their particular industry. The advantage will by no means be limited to the greater efficiencies. The real goal will be the many new business models and revenue streams the digital supply chain will open up.

Start with pilot projects. Use them to establish proof of concept and demonstrate business value. Not every project will succeed, but they will all help you learn the approach that works for your company. With early successes, you can also gain buy-in from the organization, and secure funding for a larger rollout. For the early pilots, define a relatively narrow initial scope, but incorporate the end-to-end concept of Supply Chain 4.0- from materials to the customer delivery (and services after the sale). Design pragmatically to compensate for standards or infrastructure that doesn't yet exist. Collaborate with digital leaders outside your company's boundaries; work with startups, universities, or industry organizations to accelerate your digital innovation. 
As the name suggests Supply Chain 4.0 is a revolution, meaning the changes are still ongoing and there is a lot to be learned. Industries and companies that are moving first in the marketplace, however, stand the chance of gaining competitive advantages ahead of their competitors which could, in turn, lead to an increase in market share.

Changed Management Culture If a highly influential factor exists, then it will influence every area analyzed here toward the same direction. A management culture, if it prevails, works as that factor. And in fact, the implementation of this management culture is a prerequisite for taking maximum advantage of Industry 4.0. In a company that builds such an entrepreneurial and collaborative culture across the whole organization, Supply Chain 4.0 would not be considered a disruptive innovation. Moreover, through the evolutionary process of learning by doing, the company that implements some of these emerging technologies will gradually acquire the necessary digital know-how and market responsiveness to achieve a highly competitive position in a global environment that is characterized by exponentially increasing complexity and large, rapid changes. These companies will become true order-winners by putting into practice Supply Chain Management 4.0: the high-profile management culture that will successfully integrate SCM and Industry 4.0.

As Supply Chain 4.0 takes hold around the world, emerging nations probably have the most to gain. They can leverage digitization to gain efficiency in their horizontal integration, working with the global manufacturers to whom they supply all manner of raw materials, parts, and components. The more closely they align with the platforms of Industry 4.0, the more potential customers they will be able to reach.

Supply Chain 4.0 in Industry (USE CASES) Here's How Sanofi is Embracing Supply Chain 4.0 and Blockchain Technology in its Supply Chain The face of manufacturing and supply chains is changing as the fourth industrial revolution continues to gather steam. Exciting new technology is being incorporated at all levels of the logistics industry, and global biopharmaceutical brand Sanofi is no different. The Supply Chain 4.0 as it's known, refers to the fourth major paradigm shift which has occurred in production. The fourth industrial revolution is seeing those same processes now brought online, as digital technology such as the Internet of Things connects machines and computers like never before. Supply Chain 4.0 for Logistics The most clear and obvious application of Supply Chain 4.0 technology to the logistics business is Internet of Things technology. With connected sensors installed in trucks and shipping containers, real time data can be fed back to base allowing for monitoring and maintenance. Data can include temperature, motion, weight, and more. With many medical products requiring carefully maintained conditions this is of particular importance in this industry. At the other end of this system is the enormous amount of data which is generated by this type of technology. With the employment of data scientists, the analysis of this data can lead to improvements and efficiencies throughout the logistics chain. Shipping times can be optimised by identifying where slowdowns and delays occur.

"The new age of IoT is an exciting one for logistics," writes Victoria Greene for Iotforall.com. "With so many opportunities to streamline chain management and optimize every element of production, the possibilities really are endless. Customers too will see the benefits with easy-to-track deliveries, an improved shopping experience and greater rewards. All in all, the dawn of the IoT will bring with it many changes in the future - we're just getting warmed up!" Blockchain Another new technology which Sanofi is actively pursuing is blockchain. For the uninitiated, blockchain technology allows anonymous parties to carry out secure and private transactions without the need for a central agency to verify them. While the term "transaction" is often used, blockchain can be used for any kind of data exchange, not just financial ones. Each packet of information (or block) which is added to the blockchain contains data about all the previous entries. This means that any attempt to manipulate the chain will become immediately apparent, as it will also change information in every link. It's this transparency which grants blockchains their much-valued security, and removes the need for the central authority.

In pharmaceutical supply chains, blockchain has many potential applications - from ensuring the security of medical devices to the safe transfer of medical records, facilitating more efficient clinical trials, and getting speedier approval of new drugs. The technology can also increase cost transparency - essential in pharma - and prevent counterfeit drugs from entering the supply chain. With an estimated one in ten medical products in developing countries being counterfeited, the problem is a serious one. With blockchain, however, a system can be developed which allows medical solutions to be tracked from production to patient. Therefore, it would be nearly impossible to sneak fake medicine into the supply chain undetected, as it would lack the necessary blockchain authorisation. "Blockchain is really about industrialising trust," said Sanofi's Chief Data Officer, Milind Kamkolkar. "We're seeing consortiums forming already to deal with this issue." Supply Chain 4.0 and blockchain technology were hot topics at Logi Pharma 2019, in April, at the 2m2c Montreux Music \& Convention Canter, Switzerland.

\section{CONCLUSION}

Supply Chain 4.0 in process has evolved various new approaches to product distribution, Procurement, Fulfilment, transportation and customer experience. This would reduce the delivery time, manage inventories without any loss in the process. Digitization has enabled the use of advanced forecasting approaches, such as predictive analytics of internal data (e.g., demand) and external data e.g., market trends etc., to make sure the 
consumers are supported with their demands and fulfilled before time. Latest Technology driven younger talent and Blockchain, AI and Machine Learning bringing about more intelligent analytics ,Spend Analysis Software ,Data Driven Logistics, Drone Technology, Perfect Order Deliveries , Agile Logistics.

Analytics can yield insights that help you reshape your operational designs. For example, analysis of your customers' daily and seasonal use of machinery can help you improve production schedules. Data about employee recruiting can help you predict your next talent shortfalls. Production data can illuminate opportunities to eliminate downtime or speed up throughput. Analytics can also help you balance trade-offs: for instance, the data might help an oil company decide to place a refinery offshore, even though the costs are higher, because it will yield more uptime and thus more profits.

With the dawn of the digital supply chain, a transparent visibility is encouraged for the needs and the challenges in the process. Supply and demand in an integrated part of the market. Whenever a demand is made, the market fulfils those demands through effective supplies as and when needed. If at any point supply is not met by the customers' demand, signals will originate at any point and travel immediately throughout the network to keep the situation communicative anywhere across the world. Low levels of a precarious raw material, the shutdown of a major plant, a sudden increase/decrease in customer demand - all such information will be visible throughout the system day and night, in real time. Such is the expertise of digitization in supply chain market. Where an advance information and forecasting can let market players and consumers plan.

There are three key main enablers of transformation into digital supply chain: a clear strategy, new competencies, and an advanced environment. Digital supply chain starts with an understanding of the current operation's digital waste, the lack of knowledge often leads to a failed road and thus it's important to encapsulate the technological advances. Capabilities regarding digitization then need to be created with planning. The final requirement is the implementation of an advanced technological approach. This altogether combines the advances that lead to digitally equipped innovative environment ready to penetrate any segment in and out - All for the end consumers.

As your company becomes active in Industry 4.0, you'll find the benefits go far beyond extending your digital reach or selling new types of products and services. It will establish your company, your employees, and your entire ecosystem of suppliers, partners, distributors, and customers as a fully interconnected, integrated digital network, linked to other networks around the world.

But if companies are to make the digital supply chain - or perhaps more properly, the digital supply chain ecosystem a reality, they can't just gather technologies and build capabilities. They must also find people with the right skills, and manage the shift to a culture that's willing to carry out the effort. In other words, they must transform their entire organization.

\section{REFERENCES}

1. Envisioning SCM 4.0: The view from Japan - Manufacturing .... (1970) Retrieved on September 9, 2019, from https://www.supplychainquarterly.com/topics/Manufacturing/20161021 -envisioning-scm-40the-view-from-japan/.

2. Here's How Sanofi is Embracing Supply Chain 4.0and Blockchain .... (1970). Retrieved on September 9, 2019, from https://logipharmaeu.wbresearch.com/sanofi-embracing-industry-4-bloc kchain-technology-supply-chain-strategy-ty-u.

3. Supply Chain 4.0. (1970). Retrieved on September 9, 2019, from https://en.wikipedia.org/wiki/Industry_4.0.

4. Is Your Supply Chain Ready for Industry 4.0? . (1970). Retrieved on $\begin{array}{ll}\text { September } & 9,\end{array}$

5. Kari Miller. (1970). The Impact of Industry 40 on Quality Management Systems. Retrieved on September 9, 2019, from https://www.pilgrimquality.com/blog/smart-quality-management-impact -industry/.

6. PricewaterhouseCoopers. (1970). Industry 4.0: How digitization makes the supply chain more efficient .... Retrieved on September 9, 2019, from https://www.strategyand.pwc.com/gx/en/insights/digitization-more-effici ent.html.

7. Reinhard Geissbauer, Jesper Vedsø, and Stefan Schrauf. (1970). A strategist's guide to Industry 4.0. Retrieved on September 9, 2019, from https://www.strategy-business.com/article/A-Strategists-Guide-to-Indust ry-4.0?gko=a2260.

8. https://www.finoit.com. (1970). Digital Supply Chain in Industry 4.0. Retrieved on September 9, 2019, from https://www.finoit.com/blog/digital-supply-chain-in-industry4-0/.

9. Advanced Manufacturing | ACTPHAST 4.0. (2019). Retrieved on September 9, 2019, from https://actphast.eu/application-domain/advanced-manufacturing.

10. Cybersecurity is a key enabler for Supply Chain 4.0adoption â€” ENISA. (2019). Retrieved on September 9, 2019, from https://www.enisa.europa.eu/news/enisa-news/cybersecurity-is-a-key-en abler-for-industry-4-0-adoption.

11. Digital supply chain tech vital for auto companies. (2019). Retrieved on September 9, 2019, from http://www.newindianexpress.com/business/2019/aug/02/digital-supplychain-tech-vital-for-auto-companies-2012985.html

12. Digital supply chain tech vital for auto companies. (2019). Retrieved on $\begin{array}{llll}\text { September } & 9, & \text { 2019, from }\end{array}$ http://www.newindianexpress.com/business/2019/aug/02/digital-supplychain-tech-vital-for-auto-companies-2012985.html

13. Digital technology and the new supply chain revolution. (2019). Retrieved on September 9, 2019, from https://360.fmlogistic.com/retail-revolution/the-new-supply-chain-revolu tion-3/.

14. Envisioning SCM 4.0: The view from Japan â $€$ “ Manufacturing ... (2019). Retrieved on September 9, 2019, from https://www.supplychainquarterly.com/topics/Manufacturing/20161021 -envisioning-scm-40the-view-from-japan/.

15. Guest Opinion: Procurement 4.0 â $€$ “" The Digital Makeover. (2019). Retrieved on September 9, 2019, from https://www.scmr.com/article/guest_opinion_procurement_4.0_the_digi tal_makeover.

16. Has the conventional Factory Worker been able to leverage Industry .... (2019). Retrieved on September 9, 2019, from https://throughput.world/has-the-conventional-factory-worker-been-ableto-leverage-industry-4-0-advantages-yet/.

17. Here's How Sanofi is Embracing Supply Chain 4.0and Blockchain ... (2019). Retrieved on September 9, 2019, from https://logipharmaeu.wbresearch.com/sanofi-embracing-industry-4-bloc kchain-technology-supply-chain-strategy-ty-u.

18. How digitization changed the nature of supply chain management .... (2019). Retrieved on September 9, 2019, from https://www.supplychaindive.com/news/supply-chain-skills-digitizationcost-revenues-profession/437102/.

19. Supply Chain 4.0|| Supercharg3d. (2019). Retrieved on September 9 2019, from http://www.supercharg3d.com/tag/industry-4-0/.

20. Is Your Supply Chain Ready for Industry 4.0?. (2019). Retrieved on September 9, 2019, from 
https://blog.arkieva.com/supply-chain-ready-for-4-0/.

21. Life Sciences Advisory Services. (2019). Retrieved on September 9, 2019, from https://www.tcs.com/life-sciences-advisory-services.

22. Master Class in Inventory Management in Supply Chain 4.0Working .... (2019). Retrieved on September 9, 2019, from https://www.a-star.edu.sg/kto/Master-Classes/Inventory-Management-in -I4-Working-Enviroment.

23. Pragmatic real. (2019). Retrieved on September 9, 2019, from https://blog.bosch-si.com/industry40/pragmatic-real-time-logistics-a-new -material-flow-paradigm/.

24. Revolution in End. (2019). Retrieved on September 9, 2019, from https://blog.kinaxis.com/2016/10/changing-role-end-end-supply-chainmanagement/.

25. Supply Chain 4.0 and industry of the future. (2019). Retrieved on September 9, 2019, from https://www.mobility-work.com/blog/on-the-way-to-supply-chain-4-

26. What is Pharma 4.0? (2019). Retrieved on September 9, 2019, from https://www.industryofthingsvoice.com/what-is-pharma-4-0-insights-int o-the-paradigm-shift-written-by-christian-woelbeling-chairman-ispe-spec ial-interest-group-pharma-4-0/.

27. Brian Hoey. (2019). How to Make the Most of the Supply Chain 4.0Supply Chain. Retrieved on September 9, 2019, from https://blog.flexis.com/industry-4.0-supply-chain.

28. Sudip Singh. (2019). Demystifying Supply Chain 4.0and the industrial internet of things. Retrieved on September 9, 2019, from https://yourstory.com/2017/07/demystifying-industry-industrial-internetof-things.

29. https://www.finoit.com. (2019). Digital Supply Chain in Industry 4.0. Retrieved on September 9, 2019, from https://www.finoit.com/blog/digital-supply-chain-in-industry4-0/. 